\title{
Tendência da mortalidade relacionada à doença de Chagas, Estado de São Paulo, Brasil, 1985 a 2006: estudo usando causas múltiplas de morte
}

\author{
Augusto Hasiak Santo ${ }^{1}$
}

Como citar

Santo AH. Tendência da mortalidade relacionada à doença de Chagas, Estado de São Paulo, Brasil, 1985 a 2006: estudo usando causas múltiplas de morte. Rev Panam Salud Publica. 2009;26(4):299-309.

RESUMO Objetivo. Estudar a tendência da mortalidade relacionada à doença de Chagas informada em qualquer linha ou parte do atestado médico da declaração de óbito.

Métodos. Os dados provieram dos bancos de causas múltiplas de morte da Fundação Sistema Estadual de Análise de Dados de São Paulo (SEADE) entre 1985 e 2006. As causas de morte foram caracterizadas como básicas, associadas (não-básicas) e total de suas menções.

Resultados. No período de 22 anos, ocorreram 40002 óbitos relacionados à doença de Chagas, dos quais 34917 (87,29\%) como causa básica e 5085 (12,71\%) como causa associada. Foi observado um declínio de 56,07\% do coeficiente de mortalidade pela causa básica e estabilidade pela causa associada. O número de óbitos foi $44,5 \%$ maior entre os homens em relação às mulheres. O fato de $83,5 \%$ dos óbitos terem ocorrido a partir dos 45 anos de idade revela um efeito de coorte. As principais causas associadas da doença de Chagas como causa básica foram as complicações diretas do comprometimento cardíaco, como transtornos da condução, arritmias e insuficiência cardiaca. Para a doença de Chagas como causa associada, foram identificadas como causas básicas as doenças isquêmicas do coração, as doenças cerebrovasculares e as neoplasias. Conclusões. Para o total de suas menções, verificou-se uma queda do coeficiente de mortalidade de 51,34\%, ao passo que a queda no número de óbitos foi de apenas 5,91\%, tendo sido menor entre as mulheres, com um deslocamento das mortes para as idades mais avançadas. A metodologia das causas múltiplas de morte contribuiu para ampliar o conhecimento da história natural da doença de Chagas.

Palavras-chave Doença de Chagas; causas múltiplas de morte; mortalidade; processamento automatizado de dados; Brasil.

A doença de Chagas é uma infecção parasitária crônica causada pelo Trypanosoma cruzi. Entre 20 e 30\% dos indivíduos infectados manifestam a doença, caracterizada por cardiopatia grave, megaesôfago ou megacólon. Trata-se de

1 Universidade de São Paulo, Faculdade de Saúde Pública, Departamento de Epidemiologia, São Paulo, Brasil. Correspondência: Rua Pará 21, apt. 62, CEP 01243-020, São Paulo, SP, Brasil. Fone: +55-11-3258.4760; fax: +55-11-3259.2612; e-mail: auhsanto@usp.br uma zoonose complexa, que inclui numerosos vertebrados como reservatórios e insetos triatomídeos que participam da cadeia de transmissão, o que torna impossível a sua erradicação. Estima-se que a doença de Chagas continue a representar uma ameaça à saúde de 28 milhões de pessoas na América Latina. Nessa região, de 9,8 a 15 milhões de indivíduos continuam infectados (1-4), sendo 3,5 milhões no Brasil (5).
A estratégia para a eliminação da doença de Chagas baseia-se no controle de vetores, na triagem sistemática de doadores de sangue nos países endêmicos, no diagnóstico e tratamento da transmissão congênita e no tratamento de crianças infectadas e de casos agudos (2). Essas medidas de intervenção contribuíram para interromper a transmissão em áreas consideráveis e são usadas para monitorar o impacto do controle da 
doença de Chagas (6). Brasil, Chile e Uruguai foram certificados livres da transmissão pelo Triatoma infestans, principal vetor domiciliado nesses países $(2,4,7)$. No Estado de São Paulo, a transmissão vetorial da doença de Chagas foi interrompida no início da década de 1970 e a vigilância entomológica continuada não tem detectado colônias intradomiciliares de triatomídeos associados à infecção pelo Trypanosoma cruzi (8-10).

As estatísticas de mortalidade dos países não têm sido muito usadas para descrever o impacto das medidas de controle da doença de Chagas. Esse fato reflete-se no Relatório Técnico sobre o Controle da Doença de Chagas, promovido pela Organização Mundial da Saúde (OMS), e no Consenso Brasileiro em Doença de Chagas, nos quais não ocorre a descrição de dados sobre mortalidade $(1,11)$. Recentemente, entretanto, o Relatório do Grupo Científico de Trabalho sobre Doença de Chagas apresentou estimativas declinantes da tendência das mortes anuais, de 45000,21000 e 12 500, respectivamente, em 1990, 2000 e 2006, como resultado da interrupção da transmissão nas regiões endêmicas (2). Entretanto, são escassos no Brasil os estudos sobre a mortalidade relacionada à doença de Chagas na população (12).

Como descrito anteriormente (13), as estatísticas nacionais de mortalidade têm origem nas declarações de óbito (14-16). Os dados demográficos e médicos da declaração de óbito são consolidados para todo o país pelo Ministério da Saúde (17). Dentre esses dados, sobressaem-se as causas de morte, das quais a causa básica é conceituada como a) a doença ou lesão que iniciou a cadeia de acontecimentos patológicos que conduziram diretamente à morte, ou b) as circunstâncias do acidente ou violência que produziram a lesão fatal (18).

Objetivando prevenir a morte, as estatísticas primárias de mortalidade são apresentadas tradicionalmente segundo a causa básica. Entretanto, nas últimas décadas, aumentou a demanda pela apresentação de todas as causas de morte informadas nas declarações de óbito, e não apenas da causa básica. Essas estatísticas, ditas por causas múltiplas, podem fornecer informações sobre todo o processo letal que culminou com a morte, oferecendo novos elementos e perspectivas para a sua prevenção $(19,20)$. Dados relativos às causas múltiplas de morte estão disponíveis no Estado de São Paulo desde
1983, e no Brasil, desde 1999 (21, 22). No Brasil, em 2003, nos estados com dados confiáveis sobre causas múltiplas, ocorreram 5938 óbitos relacionados à doença de Chagas, dos quais $4931(83,04 \%)$ como causa básica de morte (21).

Este trabalho objetivou estudar a tendência da mortalidade relacionada à doença de Chagas no Estado de São Paulo entre 1985 e 2006 por meio da metodologia das causas múltiplas, considerando todos os óbitos nos quais a doença de Chagas foi informada em qualquer linha ou parte do modelo internacional de atestado médico de causa de morte da declaração de óbito, e não apenas quando identificada como causa básica de morte.

\section{MATERIAIS E MÉTODOS}

Os dados sobre os óbitos provieram dos bancos anuais incluindo causas múltiplas de morte da Fundação Sistema Estadual de Análise de Dados (SEADE), órgão de governo responsável pela elaboração de estatísticas vitais em São Paulo. Foram estudados todos os óbitos nos quais a doença de Chagas foi mencionada em qualquer linha ou parte do modelo internacional de atestado médico de causa de morte (atestado médico da declaração de óbito) (18), independentemente de ser qualificada como causa básica ou causa associada (não-básica) de morte $(19,20)$. As complicações da causa básica (parte I do atestado médico) e as causas contribuintes (parte II do atestado médico) foram consideradas conjuntamente como causas associadas de morte (19). Do mesmo modo, foram utilizadas as estimativas populacionais preparadas pela Fundação SEADE para o período entre 1985 e 2006. As classes dos grupos etários de 75 anos ou mais foram criadas aplicando-se as proporções correspondentes verificadas no censo do ano 2000 no Estado de São Paulo.

Os registros dos bancos de óbitos reproduziam em seus campos os respectivos itens da declaração de óbito em uso no Brasil. Neste trabalho houve necessidade de modificar a estrutura dos registros incluídos nos bancos de dados. Entre 1985 e 2006, vários campos sofreram mudanças, além de duas revisões da Classificação Internacional de Doenças (CID) estarem em vigor no período. As características dos campos, tais como designação, tamanho e estrutura de códi- gos, foram uniformizadas para consolidar os arquivos anuais do período de 22 anos e permitir o processamento. Campos auxiliares foram criados para facilitar a manipulação dos dados, dentre os quais um campo para conter um string, formado por uma cadeia única de caracteres constituída pelos códigos registrados nas linhas (a), (b), (c), (d) da parte I e da parte II do atestado médico da declaração de óbito.

As causas de morte foram processadas automaticamente entre 1985 e 1995 pelo programa Automated Classification of Medical Entities (ACME) $(19,20,22-25)$ e entre 1996 e 2005 pelo programa Declarações de Óbito de São Paulo (DOSP) (26), uma adaptação para processamento em lote do programa Seleção de Causa Básica (SCB) (22). Os dados sobre as causas associadas de morte do ano de 1996 são parciais devido ao início de uso do DOSP pela Fundação SEADE a partir do mês de abril desse ano. $O$ processamento automático é realizado por meio de algoritmos e tabelas de decisão que incorporam as regras de mortalidade da OMS e as relações etiológicas entre as causas de morte. As tabelas de decisão do ACME são consideradas como padrões de facto para o processamento automático de causas de morte (27). Foram revistos os registros em que a doença de Chagas foi mencionada no atestado médico mas não foi selecionada como causa básica. Essa revisão objetivou assegurar a propriedade pela identificação da doença de Chagas como causa básica de morte pelo processamento automático (19, 22-26, $28,29)$. A revisão dos registros de óbito em que a doença de Chagas havia sido identificada como causa associada permitiu a reclassificação de 2638 dessas mortes como causa básica. A doença de Chagas é uma causa de morte rara, tendo ocorrido apenas 13 óbitos como causa básica nos Estados Unidos entre 1999 e 2004 (30), razão pela qual as tabelas de decisão para processamento automático não previam todas as relações etiológicas possíveis de ocorrer. Desse modo, o sistema gerava eventualmente uma complicação em lugar da doença de Chagas como causa básica. Portanto, o número de óbitos por doença de Chagas como causa básica neste estudo é superior ao apresentado nos bancos originais de mortalidade da Fundação SEADE.

Neste estudo, com a finalidade de reconstituir o processo mórbido que conduziu ao óbito, todas as causas de morte 
informadas no atestado médico foram consideradas, mesmo as causas mal definidas e aquelas caracterizadas pela OMS como modos de morrer (18). A doença de Chagas, como causa de morte, correspondeu às formas clínicas incluídas nas subcategorias 086.0 (doença de Chagas com comprometimento cardíaco), 086.1 (doença de Chagas com comprometimento de outro órgão), 086.2 (doença de Chagas sem menção de órgão comprometido) e 086.9 (tripanossomíase não especificada) da Nona Revisão da Classificação Internacional de Doenças (CID-9) e na categoria B57 (doença de Chagas), compreendendo todas as subcategorias incluídas, da Décima Revisão da Classificação Estatística Internacional de Doenças e Problemas Relacionados à Saúde (CID-10) $(31,32)$. Tabelas de equivalência foram preparadas para comparar a tendência das causas de morte relacionadas à doença de Chagas entre essas duas revisões $(33,34)$. A diferença na estrutura de códigos entre a CID-9, numérica, usada até 1995, e a CID-10, alfanumérica, usada a partir de 1996, determinou o processamento dos dados por meio de programas adaptados a essas estruturas e, por consequência, a criação dos seguintes períodos de tempos para o estudo da tendência da mortalidade relacionada à doença de Chagas: 1985 a 1990, 1991 a 1995, 1996 a 2000 e 2001 a 2006. As expressões "óbitos por" ou "devido a" determinada afecção referem-se à causa básica de morte; as expressões "óbitos com menção de" e "mortalidade relacionada à doença de Chagas" correspondem à presença dessa afecção informada quer como causa básica ou associada (nãobásica) no atestado médico. As causas de morte usadas no estudo foram aquelas mencionadas originalmente no atestado médico, qualificadas internacionalmente como entity axis codes, definidas e apresentadas por meio da estrutura e das rubricas da CID $(19,35)$.

Foram estudadas as distribuições das variáveis sexo, idade ao morrer, causas básica e associada de morte, número médio de causas informadas por declaração de óbito e regiões de residência do falecido e de ocorrência do óbito, por meio de coeficientes, proporções e tendência histórica. As variáveis médicas e demográficas foram processadas pelos programas dBASE III Plus, versão 1.1, Epi Info, versão 6.04d, e Excel 2007. O programa Tabulador de Causas Múltiplas (TCM), com versões para a CID-9 e
CID-10 (TCM-9, v. 4.0, e TCM-10, v. 2.2) foi usado para a apresentação das causas associadas e do número médio de causas por declaração de óbito (36). Para a apresentação das causas associadas nos óbitos em que a doença de Chagas foi identificada como causa básica foram preparadas listas especiais que evidenciassem tanto as causas envolvidas na sua história natural quanto as mencionadas com maior frequência. A duplicação ou multiplicação de causas de morte foi eliminada para sua apresentação por meio de listas abreviadas. Como o número de causas depende da amplitude da classe (subcategoria, categoria, agrupamento ou capítulo da CID-9 e CID-10), apenas uma causa foi computada se duas (ou mais) causas mencionadas no atestado médico estivessem incluídas numa mesma classe $(19,36)$. Os códigos secundários em asterisco que descreviam as manifestações clínicas ou complicações da doença de Chagas foram eliminados das listas abreviadas para apresentação de causas associadas. Essa eliminação foi necessária pois tais códigos, inválidos para a apresentação de causas de morte, foram usados experimentalmente nos Estados Unidos e no Brasil (São Paulo) no processamento automático das causas de morte entre os anos de 1985 e 1989. O programa Separador de Registros de Mortalidade (SRM_DBF), com respectivas versões para a CID-9 e CID-10 (v. 1.1, 2007, e v. $3.1,2002$, DATASUS), foi usado para a recuperação de registros em que houvesse interesse em determinadas causas associadas de morte.

Coeficientes de mortalidade (por 100000 habitantes) foram calculados para os óbitos em que a doença de Chagas foi identificada como causa básica ou associada, bem como pelo total de suas menções segundo anos, subperíodos e período total entre 1985 e 2006. Para o cálculo dos coeficientes, em cada um dos subperíodos estudados, o número de óbitos ocorridos no subperíodo foi dividido pela soma das respectivas populações anuais correspondentes; um procedimento análogo foi usado para o período total de 22 anos. O Programa para Análise Epidemiológica de Dados Tabulados (Epidat), versão 3.1, foi usado para padronizar, pelo método direto, os coeficientes de mortalidade anuais, dos subperíodos e totais, por idade e pela população do Brasil de 2000.

O estudo do efeito de coorte ou de gerações sobre a mortalidade relacionada à doença de Chagas foi realizado por meio da formação de grupos etários e períodos de igual amplitude. Foram criados 16 grupos etários quinquenais a partir de 0 a 4 anos até 75 a 79 anos, além de um grupo idoso de 80 anos ou mais, assim como quatro períodos de tempo compreendidos entre 1985 e 1989 e 2000 e 2004, atingindo o mínimo útil de 20 anos para esse tipo de estudo (37). Os óbitos com idade ignorada foram eliminados no cálculo da idade ao morrer e dos coeficientes específicos por idade e sexo. $\mathrm{O}$ número médio de causas informadas por declaração de óbito é apresentado com o respectivo desvio-padrão (DP).

\section{RESULTADOS}

Entre 1985 e 2006 ocorreram no Estado de São Paulo 40002 óbitos relacionados à doença de Chagas, dos quais 34917 $(87,29 \%)$ como causa básica e 5085 $(12,71 \%)$ como causa associada de morte, resultando em números médios anuais, respectivamente, de 1818,1587 e 231 óbitos. O número de óbitos foi $44,5 \%$ maior nos homens em relação aos óbitos entre as mulheres, principalmente quando a doença de Chagas se apresentou como causa básica $(47,7 \%)$. A maior proporção dos óbitos $(83,5 \%)$ ocorreu a partir dos 45 anos de idade, particularmente entre 55 e 64 anos como causa básica $(24,7 \%)$ e entre 65 e 74 anos como causa associada (tabela 1). Em números absolutos, as mortes como causa básica passaram de 1692 em 1985 a 1428 em 2006, correspondendo a uma diminuição de $15,6 \%$, ao passo que, como causa associada, foram respectivamente 168 e 322 nesses anos, configurando um aumento de $91,67 \%$, o que resulta numa queda total de apenas 5,91\%.

\section{Mortalidade proporcional}

A mortalidade proporcional pela doença de Chagas reproduz o padrão observado com o número de óbitos. Como causa básica verificou-se uma queda progressiva da mortalidade proporcional nos subperíodos, de 0,90 a $0,60 \%$, e um aumento como causa associada de 0,10 para 0,12\% entre 1985 e 1990 e 2001 e 2006 (tabela 1). Esses valores dos subperíodos sintetizam as tendências ocorridas entre os anos de 1985 e 2006, respectivamente uma queda como causa básica, de 0,93\% a $0,59 \%$, e um aumento como causa associada, de $0,09 \%$ a $0,13 \%$. 


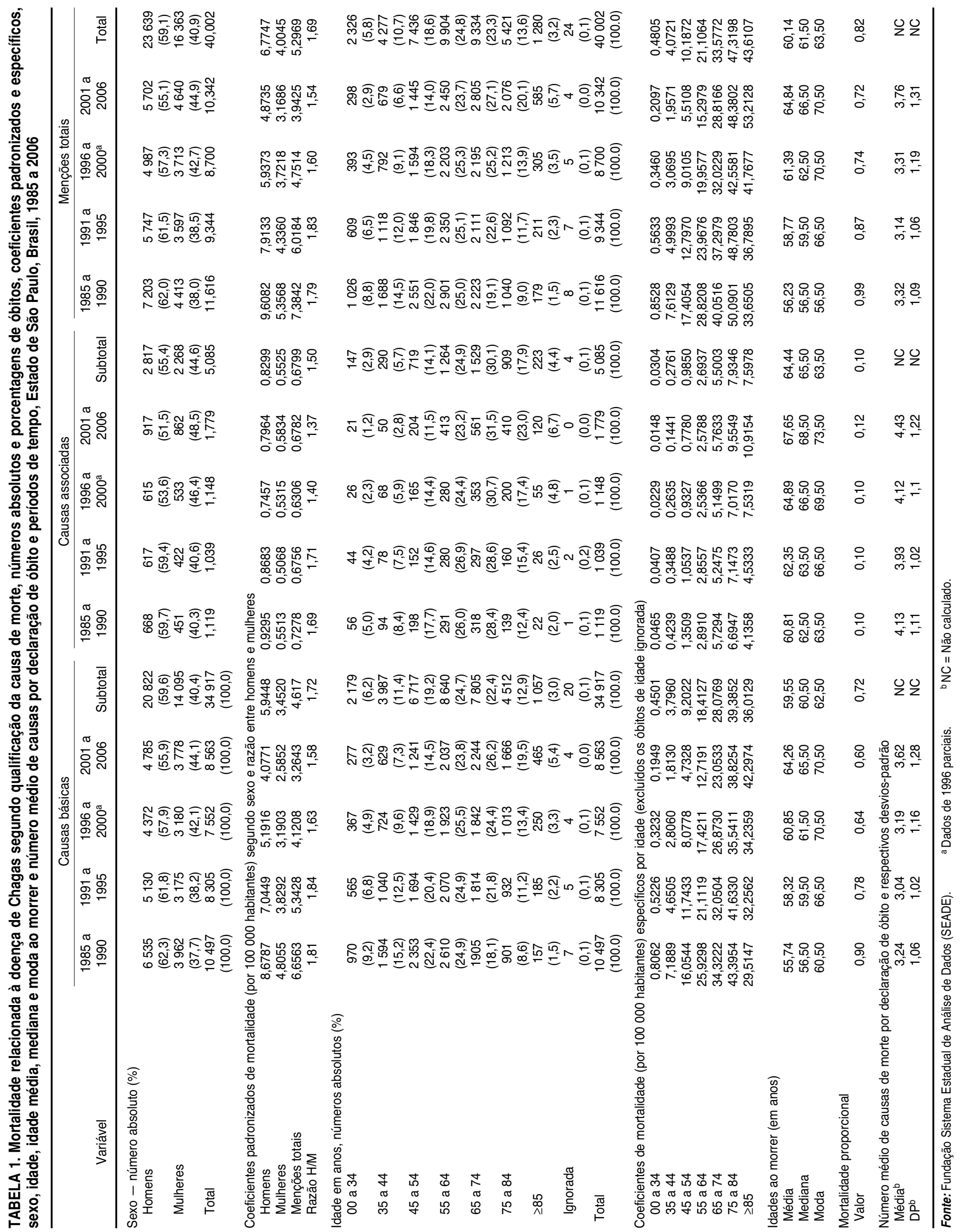




\section{Coeficientes de mortalidade}

No período de 22 anos, o coeficiente de mortalidade padronizado pelo total de menções da doença de Chagas diminuiu $51,34 \%$, passando de 7,60 a 3,70 óbitos por 100000 habitantes entre os anos de 1985 e 2006. Essa queda foi ponderada principalmente pela diminuição correspondente de $56,07 \%$ do coeficiente pela causa básica, que passou de 6,89 a 3,02 óbitos por 100000 habitantes, enquanto o coeficiente pela causa associada permaneceu praticamente inalterado, com variação em torno de 0,68 óbitos por 100000 habitantes entre esses anos (figura 1). Essas tendências refletem-se nos coeficientes sintetizados pelos quatro subperíodos estudados (tabela 1).

\section{Coeficientes de mortalidade segundo o sexo}

Os coeficientes de mortalidade padronizados foram superiores entre os homens em relação às mulheres, observando-se que as razões correspondentes da mortalidade relacionada à doença de Chagas pelo total de suas menções foram respectivamente de 1,79, 1,83, 1,60 e 1,54 nos quatro subperíodos entre 1985 e 2006. Esse fato reflete-se nas proporções decrescentes de óbitos nos homens e crescentes nas mulheres no decorrer dos subperíodos. Consequentemente, a diminuição dos coeficientes também foi maior entre os homens $(52,89 \%)$ do que entre as mulheres $(46,46 \%)$. A razão da mortalidade entre homens e mulheres, de 1,72, foi maior nos óbitos em que a doença de Chagas apresentou-se como causa básica do que nos óbitos em que se apresentou como causa associada (razão de 1,50$)$ para o período total de 22 anos (tabela 1).

\section{Coeficientes de mortalidade segundo a idade}

Os coeficientes específicos de mortalidade foram diretamente proporcionais às idades até o grupo de 75 a 84 anos, em que os maiores valores foram atingidos, diminuindo a seguir no grupo de 85 anos ou mais, o qual apresentou o segundo maior coeficiente. A maioria dos grupos etários, para o total de menções da doença de Chagas, apresentou coeficientes decrescentes ao longo dos subperíodos. As exceções ocorreram na faixa de 75 a 84 anos, cujo coeficiente no período 2001 a 2006 foi superior ao do subperíodo anterior e, principalmente, no grupo de 85 anos ou mais, cujos coeficientes foram crescentes do primeiro ao último subperíodo. Este padrão correspondeu ao da doença de Chagas como causa básica de morte. O número médio anual de óbitos totais ao longo dos subperíodos foi decrescente até os 64 anos (1 361, 1 184, 996 e 812 óbitos por ano) e, de modo inverso, crescente nos grupos

FIGURA 1. Coeficientes padronizados de mortalidade relacionada à doença de Chagas, Estado de São Paulo, Brasil, 1985 a 2006

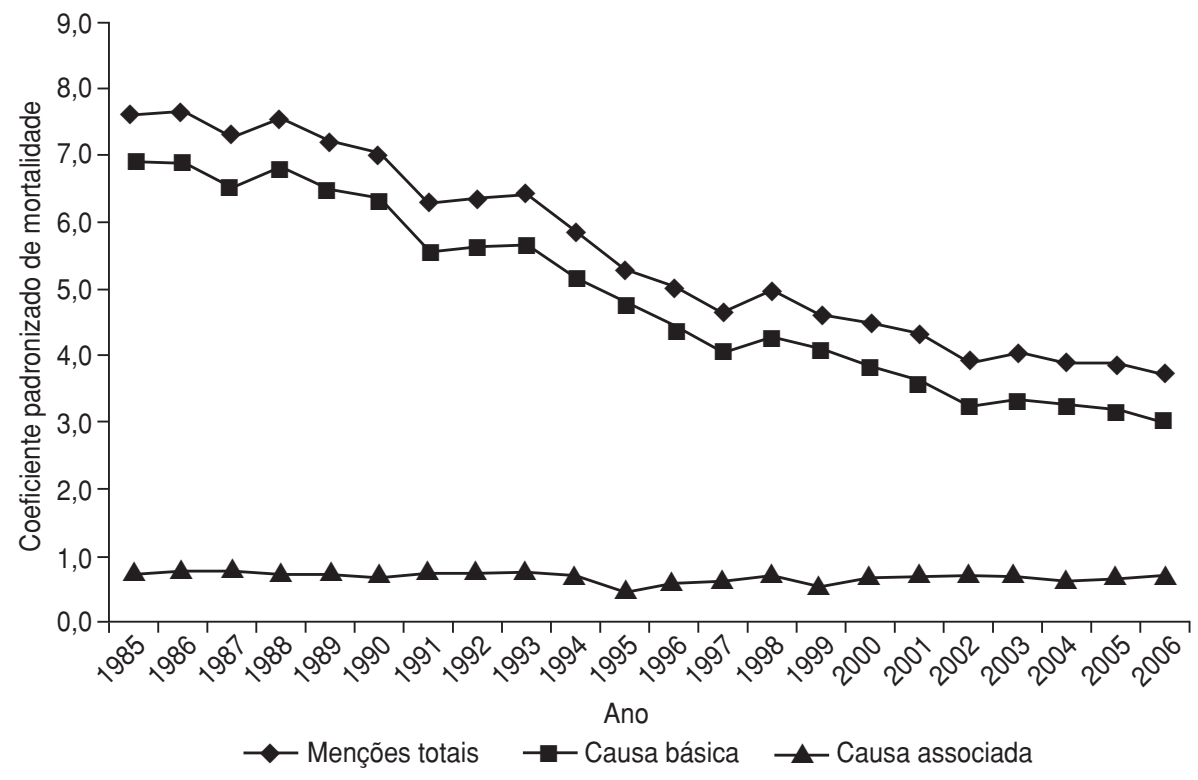

de 65 anos ou mais $(573,682,743$ e 911 óbitos por ano) (tabela 1).

\section{Média, mediana e moda da idade ao morrer}

Observou-se que as médias e medianas de idade ao morrer foram crescentes no decorrer dos quatro subperíodos. Entretanto, as modas permaneceram em 70,5 anos entre 1996 e 2006. Do mesmo modo que com os grupos etários, esse padrão reproduziu o ocorrido com os óbitos em que a doenças de Chagas apresentou-se como causa básica, notando-se, entretanto, valores mais elevados como causa associada (tabela 1). Esses indicadores foram maiores entre as mulheres do que entre os homens em todos os subperíodos; os dados consolidados da idade ao morrer para o período total de 22 anos foram, respectivamente, médias de 62,61 e 58,48, medianas de 63,50 e 59,50 e modas de 63,50 e 56,50 anos.

\section{Efeito de coorte sobre a mortalidade}

A figura 2 mostra os coeficientes de mortalidade relacionados à doença de Chagas segundo o período de nascimento e o grupo etário dos falecidos. Os grupos etários até 15 a 19 anos não foram representados, pois os respectivos coeficientes eram menores que 1 óbito por 100000 habitantes. Nota-se que as curvas dos coeficientes mais elevados correspondem aos períodos de nascimento mais antigos e aos grupos mais idosos.

\section{Coeficientes de mortalidade segundo regiões de saúde}

A figura 3 mostra os coeficientes brutos de mortalidade relacionados à doença de Chagas para os óbitos ocorridos nas 24 direções regionais de saúde (DIR) do Estado de São Paulo, calculados para o total do período entre 1985 e 2006. Os óbitos de residentes em outras unidades da federação não foram incluídos. As barras da figura são formadas pela soma dos coeficientes de mortalidade como causa básica e associada. Os valores conjuntos resultam no coeficiente de mortalidade pelas menções totais da doença de Chagas nas declarações de óbito. Verifica-se que esses valores, para o Estado de São Paulo, são respectivamente de 4,81, 0,70 e 5,51 óbitos por 100000 habitantes. Dentre as DIR 
FIGURA 2. Coeficientes de mortalidade relacionados à doença de Chagas segundo período de nascimento e idade, Estado de São Paulo, Brasil, 1985 a 2004

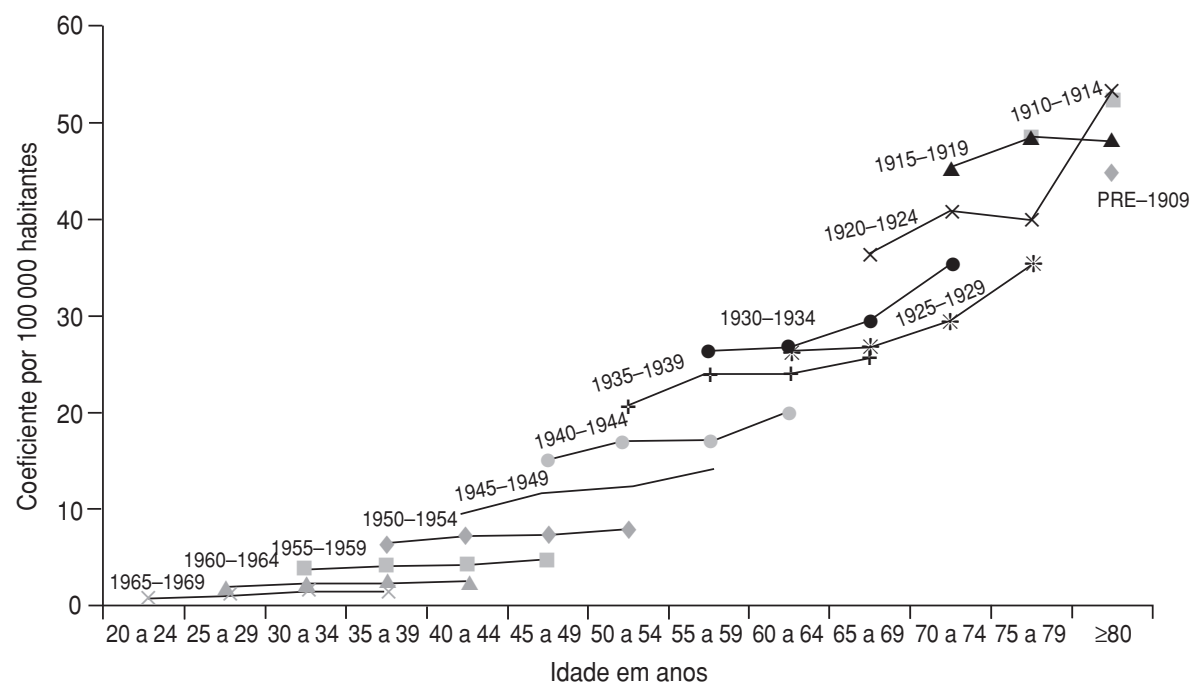

FIGURA 3. Coeficientes brutos de mortalidade por 100000 de habitantes relacionados à doença de Chagas segundo direções regionais de saúde (DIR), Estado de São Paulo, Brasil, 1985 a 2004

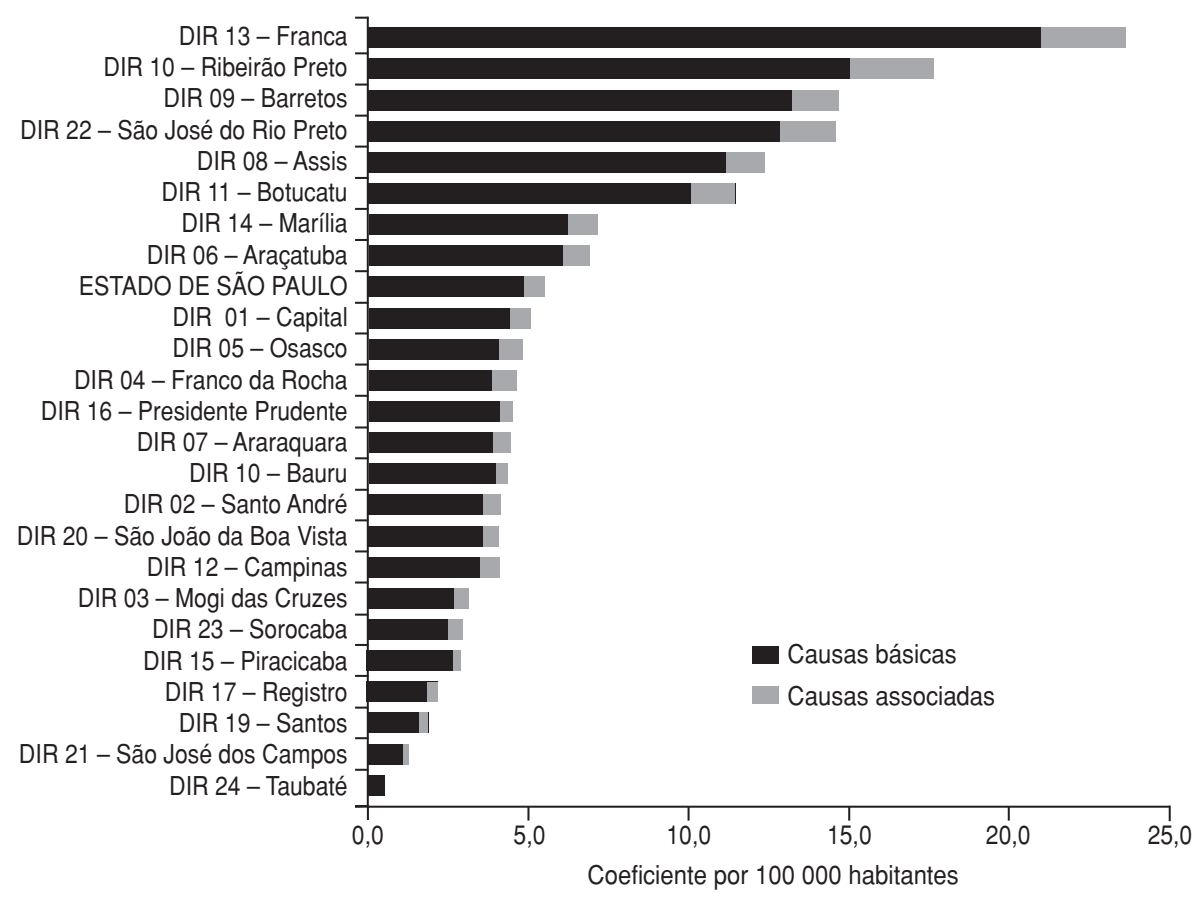

com valores superiores a 5,51 destacamse principalmente Franca, Ribeirão Preto, Barretos e São José do Rio Preto, as quais, além dos coeficientes elevados, apresentaram grande número de óbitos, respectivamente 2 666, 3 737, 1114 e 3 795, assim como as DIR da capital São Paulo e Campinas, com 10433 e 2582 óbitos, respectivamente. e 1990, observando-se uma queda no segundo subperíodo e a seguir uma tendência a aumento nos subperíodos subsequentes. Esses valores foram maiores para a doença de Chagas como causa associada do que como causa básica de morte (tabela 1).

\section{Causas associadas de morte}

A tabela 2 mostra as causas associadas (não-básicas) de morte mencionadas nas declarações de óbito em que a doença de Chagas apresentou-se como causa básica, ordenadas pela sua distribuição decrescente no primeiro período de 1985 a 1990. As complicações diretas do envolvimento cardíaco predominam, destacando-se os transtornos da condução e arritmias e a insuficiência cardíaca, ambas causas mencionadas em mais de $30 \%$ dos óbitos, seguindo-se o choque como causa terminal. Dentre as doenças do aparelho respiratório, destacam-se o edema agudo de pulmão e a embolia pulmonar. A síndrome da morte súbita foi mencionada em 2,36\% (823/39 917) dos atestados médicos entre 1985 e 2006. As causas associadas do comprometimento intestinal pela doença de Chagas, tais como o megacólon, megaesôfago e as suas complicações foram mencionadas em menor frequência. Nota-se a presença de causas de morte relacionadas às doenças crônicas do aparelho circulatório, notadamente doenças hipertensivas, isquêmicas do coração, cerebrovasculares e aterosclerose.

\section{Causas básicas com a doença de Chagas como causa associada de morte}

As causas básicas de morte naqueles óbitos em que a doença de Chagas foi identificada como causa associada, correspondentes ao total do período entre 1985 e 2006, são apresentadas na tabela 3. As doenças isquêmicas do coração, doenças cerebrovasculares e neoplasias foram responsáveis por $52,6 \%$ dessas mortes. Dentre as doenças crônicas das vias aéreas inferiores chama a atenção o elevado número de óbitos pela doença pulmonar obstrutiva crônica.

Verificou-se que o número médio de causas mencionadas por declaração de óbito foi sempre superior a três, considerando a doença de Chagas como causa básica, associada ou pelo total de suas menções. Essa média foi maior entre 1985

\section{Invasão e evasão de óbitos}

Considerando exclusivamente os óbitos relacionados à doença de Chagas de falecidos residentes no Estado de São 
TABELA 2. Causas associadas (não-básicas) de morte nos óbitos por doença de Chagas como causa básica segundo subperíodos de tempo, Estado de São Paulo, Brasil, 1985 a 2006

\begin{tabular}{|c|c|c|c|c|c|c|c|c|}
\hline \multirow[b]{3}{*}{ Causas associadas (não-básicas) de morte (CID-9) (CID-10) } & \multirow{2}{*}{\multicolumn{2}{|c|}{$\frac{1985 \text { a } 1990^{a, b}}{\text { (óbitos = } 10497 \text { ) }}$}} & \multirow{2}{*}{\multicolumn{2}{|c|}{$\frac{1991 \text { a } 1995^{a, b}}{\text { (óbitos = } 8305 \text { ) }}$}} & \multirow{2}{*}{\multicolumn{2}{|c|}{$\begin{array}{c}1996 \text { a } 2000^{\mathrm{a}, \mathrm{c}, \mathrm{d}} \\
\text { (óbitos }=7552 \text { ) }\end{array}$}} & \multirow{2}{*}{\multicolumn{2}{|c|}{$\begin{array}{l}2001 \text { a } 2006^{a, c} \\
\text { (óbitos }=8563 \text { ) }\end{array}$}} \\
\hline & & & & & & & & \\
\hline & No. & $\%$ & No. & $\%$ & No. & $\%$ & No. & $\%$ \\
\hline \multicolumn{9}{|l|}{ Transtornos da condução/Arritmias (426.0-427.9,7991) } \\
\hline$(144.0-149.9, \mathrm{R} 092)$ & 4102 & 39,08 & 3265 & 39,31 & 2994 & 39,65 & 3254 & 38,00 \\
\hline Insuficiência cardíaca (428.0-428.9) (I50.0-I50.9) & 3608 & 34,37 & 2781 & 33,49 & 2482 & 32,87 & 2949 & 34,44 \\
\hline Choque (785.5) (R57.0-R57.9) & 1436 & 13,68 & 1247 & 15,02 & 1176 & 15,57 & 1754 & 20,48 \\
\hline \multicolumn{9}{|l|}{ Outras doenças do aparelho respiratório $(510.0-516.0,516.2-516.9$, } \\
\hline 518.0-519.9, 786.0) (J80-J90, J92.0-J98.9, R09.1) & 1146 & 10,92 & 1109 & 13,35 & 1047 & 13,86 & 1507 & 17,60 \\
\hline Doenças cerebrovasculares (430-438) (I60.0-167.9, I68.0-169.8) & 588 & 5,60 & 487 & 5,86 & 501 & 6,63 & 544 & 6,35 \\
\hline Doenças isquêmicas do coração (410-414.9) (I20.0-I25.9) & 566 & 5,39 & 500 & 6,02 & 454 & 6,01 & 565 & 6,60 \\
\hline Miocardite e doenças cardíacas mal definidas (429.0-429.9) (I51.0-I51.9) & 487 & 4,64 & 405 & 4,88 & 311 & 4,12 & 500 & 5,84 \\
\hline \multicolumn{9}{|l|}{ Embolia pulmonar e outras doenças cardíacas pulmonares e } \\
\hline da circulação pulmonar (415.0-417.9) (I26.0-I28.9) & 476 & 4,53 & 319 & 3,84 & 259 & 3,43 & 256 & 2,99 \\
\hline Pneumonias (480.0-483, 485-486) (J12.0-J16.8, J18.0-J18.9) & 457 & 4,35 & 421 & 5,07 & 488 & 6,46 & 852 & 9,95 \\
\hline Cardiomiopatias $(425.0-425.5,425.9)(142.0-142.9)$ & 365 & 3,48 & 574 & 6,91 & 906 & 12,00 & 1272 & 14,85 \\
\hline Desnutrição/Caquexia (260.0-263.9, 799.4) (E40-E46, R64) & 304 & 2,90 & 279 & 3,36 & 176 & 2,33 & 233 & 2,72 \\
\hline Morte súbita de causa desconhecida (798.0-798.9) (R96.0-R96.1) & 301 & 2,87 & 227 & 2,73 & 151 & 2,00 & 144 & 1,68 \\
\hline Doenças hipertensivas (401.0-404.9) (I10-I13.9) & 290 & 2,76 & 368 & 4,43 & 418 & 5,53 & 731 & 8,54 \\
\hline Obstrução intestinal sem menção de hérnia (560.0-560.9) (K56.0-K56.7) & 261 & 2,49 & 214 & 2,58 & 245 & 3,24 & 365 & 4,26 \\
\hline \multicolumn{9}{|l|}{ Megacólon e outros transtornos funcionais do intestino (564.0-564.9) } \\
\hline (K59.0-K59.9) & 202 & 1,92 & 115 & 1,38 & 121 & 1,60 & 203 & 2,37 \\
\hline Doenças crônicas das vias aéreas inferiores (490-496) (J40-J47) & 187 & 1,78 & 196 & 2,36 & 188 & 2,49 & 237 & 2,77 \\
\hline Insuficiência renal (584.5-586) (N17.0-N19) & 187 & 1,78 & 224 & 2,70 & 284 & 3,76 & 601 & 7,02 \\
\hline Diabetes mellitus (250.0-250.9) (E10.0-E14.9) & 149 & 1,42 & 217 & 2,61 & 259 & 3,43 & 390 & 4,55 \\
\hline Aterosclerose (440.0-440.9) (I70.0-170.9) & 136 & 1,30 & 107 & 1,29 & 77 & 1,02 & 102 & 1,19 \\
\hline Septicemias (038.0-038.9) (A40.0-A41.9) & 133 & 1,27 & 133 & 1,60 & 318 & 4,21 & 710 & 8,29 \\
\hline Transtornos vasculares do intestino (557.0-557.9) (K55.0-K55.9) & 116 & 1,11 & 92 & 1,11 & 106 & 1,40 & 147 & 1,72 \\
\hline Megaesôfago e outras doenças do esôfago (530.0-530.9) (K20-K22.9) & 113 & 1,08 & 88 & 1,06 & 71 & 0,94 & 112 & 1,31 \\
\hline Peritonite (567.1-567.9) (K65.0-K65.9) & 103 & 0,98 & 89 & 1,07 & 73 & 0,97 & 132 & 1,54 \\
\hline \multicolumn{9}{|l|}{ Outras doenças do intestino $(555.0-556,562.0-562.1,565.0-566,568.0-569.9)$} \\
\hline (K50.0-K51.9, K57.0-K57.9, K60.0-K63.9, K66.0-K66.9) & 91 & 0,87 & 119 & 1,43 & 63 & 0,83 & 108 & 1,26 \\
\hline Alcoolismo (303) (F10.0-F10.9) & 86 & 0,82 & 47 & 0,57 & 41 & 0,54 & 49 & 0,57 \\
\hline Neoplasias [Tumores] (140.0-239.9) (C00.0-D48.9) & 76 & 0,72 & 54 & 0,65 & 75 & 0,99 & 92 & 1,07 \\
\hline
\end{tabular}

Fonte: Fundação Sistema Estadual de Análise de Dados (SEADE)

a Porcentagens calculadas em relação ao número de óbitos.

${ }^{b}$ Rubricas e códigos da CID-9 (1985 a 1995).

${ }^{c}$ Rubricas e códigos da CID-10 (1996 a 2004).

d Dados de 1996 parciais.

Paulo, observou-se que o Município de São Paulo, único incluído na DIR da capital, apresentou a maior invasão de óbitos de residentes em outros municípios, de 25,90\% (2 493/10 433), e a menor evasão (óbitos de residentes em São Paulo ocorridos em outros municípios), de 4,13\% (431/10 433). Por outro lado, $58,31 \%$ (1705/2 924) das invasões de óbitos na capital provieram dos municípios das DIR de Osasco, Franco da Rocha, Mogi das Cruzes e Santo André, as quais apresentaram as maiores evasões de óbitos no estado, respectivamente de $42,59 \%$ (854/2 005), 34,56\% $(113 / 327), 30,09 \%(387 / 1286)$ e $24,12 \%$ (459/1 903).

No período, verificou-se, no Estado de São Paulo, a invasão de 761 óbitos oriundos de outras unidades da fede- ração, sendo $514(67,54 \%)$ homens e $247(32,46 \%)$ mulheres, procedentes, na maioria, dos estados de Minas Gerais (449, $59,00 \%)$, Paraná $(89,11,70 \%)$ e Mato Grosso do Sul $(68,8,94 \%)$. Esses óbitos ocorreram principalmente nas DIR da capital (252/761, 33,11\%), São José do Rio Preto (186/761, 24,44\%), Ribeirão Preto $(82 / 761,10,78 \%)$ e Franca $(68 / 761,8,94 \%)$, enquanto parcela dos procedentes do $\mathrm{Pa}$ raná ocorreram nas DIR de Assis (22/89, $24,72 \%)$ e Botucatu (14/89, 15,73\%).

Por outro lado, entre 1985 e 2006 foi observada a evasão de 104 óbitos do Estado de São Paulo. Esses óbitos, resgatados por meio do Sistema de Informações sobre Mortalidade (SIM), ocorreram em outras unidades da federação e incluíam, na sua maioria, apenas a causa básica de morte, não tendo sido integrados ao estudo.

\section{DISCUSSÃO}

Este estudo procura estimular o uso das estatísticas de mortalidade na discussão sobre o impacto das medidas de controle na prevalência da doença de Chagas. Para tanto, a utilização da metodologia das causas múltiplas de morte constitui-se em fator relevante, que resgatou os óbitos nos quais a doença de Chagas não foi identificada como causa básica de morte e, por outro lado, permitiu descrever suas causas associadas, fato que ensejou, em parte, uma recomposição de sua história natural. Consequentemente, essa recomposição, por meio da análise da forma de preenchimento do atestado médico da declaração de óbito no período de 22 anos, contribuiu para o reconhecimento das entida- 
TABELA 3. Causas básicas de morte em declarações de óbito com doença de Chagas como causa associada, Estado de São Paulo, Brasil, 1985 a 2006

\begin{tabular}{lrr}
\hline \multicolumn{1}{c}{ Causas básicas de morte (CID-9) (CID-10) } & No. & $\%$ \\
\hline Doenças isquêmicas do coração (410-414.9) (I20.0-I25.9) & 1128 & 22,2 \\
Doenças cerebrovasculares (430-438) (I60.0-I67.9, I68.0-I69.8) & 856 & 16,8 \\
Neoplasias [Tumores] (140.0-239.9) (C00.0-D48.9) & 693 & 13,6 \\
Doenças crônicas das vias aéreas inferiores (490-496) (J40-J47) & 323 & 6,4 \\
Doenças hipertensivas (401.0-404.9) (I10-I13.9) & 308 & 6,1 \\
Diabetes mellitus (250.0-250.9) (E10.0-E14.9) & 204 & 4,0 \\
Outras doenças do fígado (570, 571.4-573.9) (K71.0-K76.9) & 105 & 2,1 \\
Insuficiência renal (584.5-586) (N17.0-N19) & 89 & 1,8 \\
Desnutrição (260.0-263.9) (E40-E46) & 78 & 1,5 \\
Septicemias (038.0-038.9) (A40.0-A41.9) & 71 & 1,4 \\
Doenças pelo vírus da imunodeficiência humana [HIV] (AIDS) (279.1) (B20-B24) & 68 & 1,3 \\
Doenças infecciosas intestinais (001.0-009.3) (A00.0-A09) & 44 & 0,9 \\
Tuberculose (010.0-018.9) (A15.0-A19.9) & 40 & 0,8 \\
Alcoolismo (303) (F10.0-F10.9) & 38 & 0,7 \\
Doença alcoólica do fígado (571.0-571.3) (K70.0-K70.9) & 1003 & 0,7 \\
Demais causas básicas de morte & 5085 & 19,7 \\
Total & & 100,0 \\
\hline
\end{tabular}

Fonte: Fundação Sistema Estadual de Análise de Dados (SEADE).

a Rubricas e códigos da CID-9 (1985 a 1995) e da CID-10 (1996 a 2004). Dados de 1996 parciais.

des mórbidas que não constavam de tabelas de decisão para o processamento automático de causas de morte. A incorporação dessas causas de morte ao Sistema de Seleção de Causa Básica contribuirá para o aprimoramento da identificação da correta causa básica em declarações de óbito com a menção da doença de Chagas.

Embora tenha sido observada uma pequena diminuição de óbitos entre 1985 e 2006, particularmente daqueles em que a doença de Chagas foi identificada como causa básica de morte, o número médio de óbitos assemelha-se aos achados em estudo anterior. Entre 1977 e 1986, verificou-se, no Estado de São Paulo, a ocorrência média anual de 1537 óbitos, variando entre 1455 e 1615 (38). Desse modo, nos últimos 30 anos não houve diminuição do número médio de óbitos por doença de Chagas. Esse fato reflete a cronicidade dessa enfermidade, em que a morte aguda é rara, ocorrendo normalmente em idades mais avançadas. Apesar do controle das transmissões vetorial e transfusional, estimativas por meio de modelos matemáticos mostram que os óbitos por doença de Chagas ainda devem persistir por décadas (5). A observação de ter ocorrido, no período estudado, uma diminuição dos óbitos totais de apenas $5,91 \%$, permite estranhar a estimativa de queda de $72,3 \%$ de óbitos entre 1990 e 2006 apresentada durante a reunião do grupo de trabalho científico sobre doença de Chagas, apesar da res- salva sobre serem imprecisos os dados sobre mortalidade (2).

No entanto, a tendência de queda da mortalidade relacionada à doença de Chagas foi evidenciada por meio de seus coeficientes, notadamente de 56,07\% pela causa básica de morte. Essa queda foi maior que a de $47,83 \%$ observada entre os triênios de 1981 a 1983 e 1996 a 1998 para a Região Sudeste (39), cujo valor é ponderado, em grande parte, pelos coeficientes do Estado de São Paulo. Do mesmo modo, a tendência de queda confirma-se na comparação entre a mortalidade proporcional de $0,72 \%$ nos 22 anos estudados e a de 1,04\% encontrada de 1981 a 1998 (39). Ressalve-se a influência sobre essa tendência da reduzida proporção de $12,71 \%$ de mortes em que a doença de Chagas ocorreu como causa associada, apesar do aumento de seu número e da mortalidade proporcional correspondente no período.

A observação de maior mortalidade entre os homens do que entre as mulheres é consistente com os resultados de estudos anteriores $(12,38,40)$, embora seja controverso seu valor como fator de risco (41). Porém, preocupa o inesperado achado da menor queda da mortalidade entre as mulheres, a ponto de as curvas dos respectivos coeficientes tenderem a se aproximar, fato para o qual no momento não encontramos explicação (figura não apresentada).

Os indicadores de idade ao morrer refletiram as características da cronicidade e o impacto de longo prazo das medidas para o controle e tratamento da doença de Chagas (42). Desses indicadores, a maior proporção de mortes acima de 45 anos de idade, o deslocamento das mortes para grupos etários de 65 anos ou mais e os coeficientes mais elevados nos idosos com picos entre 75 e 84 anos apontam para a maior sobrevida dos doentes. Assinale-se que esses picos foram superiores ao correspondente na faixa de 60 a 69 anos para o Brasil entre 1980 e 1995 (43). No mesmo sentido, apresentaram tendência crescente durante o período estudado as idades médias, medianas e modas ao morrer. A diminuição da mortalidade precoce tem sido atribuída aos benefícios da ausência da reinfecção, levando à redução da gravidade do quadro clínico dos infectados $(4,5,6,42)$. Ficou evidente o efeito de coorte na mortalidade relacionada à doença de Chagas, principalmente entre os idosos, em consequência da infecção pelo Trypanosoma cruzi no passado (43, 44), fato que se reitera neste estudo.

A distribuição dos coeficientes de mortalidade segundo as DIR é consistente com as descrições sobre as áreas endêmicas da doença de Chagas no Estado de São Paulo. Na década de 1950, no início das atividades de controle dos agentes vetores da doença de Chagas, todo o planalto paulista, com exceção da Grande São Paulo e do Vale do Paraíba, era considerado ampla e contínua área endêmica (8). Embora diversas ressalvas possam ser citadas, tais como o êxodo rural, a imigração para o Estado de São Paulo a partir de outras áreas endêmicas e a naturalidade dos falecidos, é válida a hipótese de que os falecidos ainda residentes nessas regiões tenham sido infectados pelo Trypanosoma cruzi há várias décadas. Essa hipótese baseia-se no sucesso da Superintendência de Controle de Endemias do Estado de São Paulo em interromper a transmissão vetorial nos primeiros anos da década de 1970 (8-10) por meio de um programa consistentemente eficaz que alcançou excelentes resultados contra a doença de Chagas $(4,42)$.

A média superior a três causas por declaração de óbito é própria dos estudos com a metodologia das causas múltiplas de morte. Ressalva-se o ganho de informação de no mínimo duas causas de morte, além da causa básica, pela consideração de todas as causas informadas na declaração de óbito. $\mathrm{O}$ aumento do 
número médio de causas verificado entre o segundo e último subperíodos estudados pode estar relacionado a uma maior atenção dada pelos médicos ao preenchimento da declaração de óbito e, a partir de 1996, a uma linha adicional na parte I do atestado médico, recomendada pela OMS com a entrada em vigor da CID-10 (18). Esse aumento também seria responsável pela maior proporção de algumas causas associadas de morte entre os subperíodos de 1985 a 1990 e 2001 a 2006.

$\mathrm{O}$ resgate das causas associadas de morte informadas no atestado médico da declaração de óbito constitui-se em recurso derivado do uso da metodologia das causas múltiplas. O conhecimento das causas associadas abre novas perspectivas para a prevenção de mortes e contribui para a discussão sobre causas com características indefinidas na história natural da doença de Chagas. As principais manifestações cardíacas e gastrointestinais mencionadas nos atestados médicos são consistentes com os relatos clínicos apresentados em nosso meio (11, 45) e em estudo análogo das causas contribuintes (46), dispensando maiores considerações. Não é o caso do acidente vascular cerebral, especialmente o isquêmico, por ser negligenciado e não reconhecido como complicação da cardiopatia chagásica (47). Uma pesquisa revelou que o diagnóstico da doença de Chagas ocorreu somente após o acidente vascular cerebral em 42,5\% dos pacientes (48). Arritmias e insuficiência cardíacas, aneurismas apicais e trombos murais, dentre outros, predispõem à embolia como causa do acidente vascular cerebral isquêmico $(47,48)$. Do mesmo modo, os dados na literatura são controversos sobre as características da associação entre a doença hipertensiva e a doença de Chagas $(49,50)$. A verificação de que essa associação determina um frequente comprometimento cardíaco de maior gravidade, principalmente nas idades mais elevadas (49), contrapõe-se ao achado de as características da hipertensão em pacientes chagásicos serem similares às observadas na população geral (50). Neste estudo, o aumento das associações com as doenças hipertensivas pode ser atribuído à mortalidade crescente nas idades mais avançadas. A morte súbita foi pouco mencionada nas declarações de óbito, embora referida em 38\% dos pacientes (45). As causas associadas consideradas terminais, como choque, pneu- monias e septicemias refletem a gravidade do processo letal relacionado à doença de Chagas.

A maior sobrevida dos pacientes chagásicos conduz a uma maior probabilidade de acúmulo de doenças crônicas como a aterosclerose, diabetes, neoplasias e doenças hipertensivas, determinando mortes relacionadas às doenças isquêmicas do coração e cerebrovasculares. Esse foi o padrão das causas básicas de morte quando a doença de Chagas se apresentou como causa associada, agravado pelo alcoolismo e suas complicações, pela tuberculose e por doenças causadas pelo vírus da imunodeficiência humana.

A invasão e a evasão de óbitos foram objeto de preocupação de um estudo anterior, o qual mostrou que $20,8 \%$ dos óbitos no Estado de São Paulo ocorreram em municípios diferentes daquele em que o falecido residia, sendo $22,8 \%$ homens e $17,4 \%$ mulheres (38). Os autores desse estudo tecem interessantes considerações sobre as razões desse fato, eventualmente relacionado à procura por parte dos pacientes de assistência médica, entretanto com prejuízo para o planejamento administrativo em saúde pública. O presente estudo mostra que a invasão de óbitos de residentes em outras unidades da federação acarreta um comprometimento adicional a esse planejamento estadual.

As limitações inerentes às estatísticas de mortalidade estão presentes neste estudo (51). Entretanto, o sub-registro de óbitos, principal limitação quantitativa, é irrelevante no Estado de São Paulo. A comparação entre os óbitos informados à Fundação SEADE e os estimados por meio de projeções demográficas, disponíveis desde 1991, mostram que, para o Estado de São Paulo, os valores superam os $100 \%$, indicando boa cobertura dos dados sobre mortalidade na população (52). As limitações qualitativas referemse à propriedade dos dados demográficos e médicos; neste estudo, especialmente à confiabilidade da doença de Chagas como causa de morte. A proporção de causas mal definidas é o indicador mais usado para avaliar a qualidade das estatísticas de mortalidade. $\mathrm{O}$ Estado de São Paulo apresenta uma das menores proporções de causas básicas mal definidas (53), atingindo, em cada um dos quatro subperíodos estudados, $5,98,6,25,6,39$ e $6,45 \%$, respectivamente, em relação ao total dos óbitos, valores in- feriores à metade dos registrados para o país $(51,53)$. O trabalho de codificação das causas de morte, atualmente realizado nos municípios de forma descentralizada, é duplicado e supervisionado por técnicos experientes na Fundação SEADE e, após o processamento automático, os bancos de dados são submetidos a rigoroso controle de qualidade (24, 25). Neste estudo, a revisão das declarações de óbito em que a doença de Chagas foi identificada como causa associada - devido a preenchimento inadequado do atestado médico ou devido a deficiências do processamento automático - permitiu a sua reclassificação como causa básica de morte.

\section{Conclusões}

Este estudo descreveu as características da mortalidade relacionada à doença de Chagas, identificada, por meio da metodologia das causas múltiplas de morte, no período de 22 anos entre 1985 e 2006. Para o total de suas menções, como causa básica ou associada, verificou-se uma queda do coeficiente de mortalidade de $51,34 \%$, ao passo que a queda do número de óbitos foi de apenas 5,91\%, queda essa cuja tendência foi menor entre as mulheres, tendo ocorrido um deslocamento das mortes para as idades mais avançadas, mostrando um evidente efeito de coorte ao atingir os infectados anteriormente aos programas de controle da transmissão nas áreas endêmicas do Estado de São Paulo. A identificação de todas as causas relacionadas à mortalidade da doenças de Chagas permitiu evidenciar afecções integrantes de sua história natural, contribuindo para orientar medidas assistenciais e para aprimorar o processamento automático de causas de morte. Espera-se que o estudo possa ainda integrar os dados de mortalidade como instrumento para a avaliação das medidas de tratamento e controle da doença de Chagas.

Agradecimentos. O autor agradece a Donna L. Hoyert, do National Center for Health Statistics, pelas indicações sobre a mortalidade relacionada à doença de Chagas nos Estados Unidos da América; e à Deise Akiko Oushiro, da Fundação Sistema Estadual de Análise de Dados, pelo levantamento sobre a evasão de óbitos por doença de Chagas no Estado de São Paulo. 


\section{REFERÊNCIAS}

1. World Health Organization. Control of Chagas disease. Second report of the WHO Expert Committee. Genebra: WHO; 2002. (Technical Report Series No 905).

2. Guhl F, Lazdins-Helds JK, eds. Reporte del grupo de trabajo científico sobre la enfermedad de Chagas. 17 a 20 de abril de 2005, Buenos Aires, Argentina. Buenos Aires: Organización Mundial de la Salud/Programa Especial de Investigaciones y Enseñanzas sobre Enfermedades Tropicales (TDR); 2007. Disponível em: http://www.who.int/tdrold/ publications/publications/swg_chagas.htm. Acessado em agosto de 2009.

3. Dias JC, Prata A, Correia D. Problems and perspectives for Chagas disease control: in search for a realistic analysis. Rev Soc Bras Med Trop. 2008;41(2):193-6.

4. Schofield CI, Jannin J, Salvatella R. The future of Chagas disease control. Trends Parasitol. 2006;22(12):583-8.

5. Massad E. The elimination of Chagas disease from Brazil. Epidemiol Infect. 2008;136(9): 1153-64.

6. Dias JC, Silveira AC, Schofield CJ. The impact of Chagas disease control in Latin America. Mem Inst Oswaldo Cruz. 2002;97(5):603-12.

7. Ramos Jr. AN, Carvalho DM. Os diferentes significados da certificação conferida ao Brasil como estado livre da doença de Chagas. Cad Saude Publica. 2001;17(6):1403-12.

8. Rocha e Silva EO, Wanderley DM, Rodrigues VL. Triatoma infestans: importância, controle e eliminação da espécie no Estado de São Paulo, Brasil. Rev Soc Bra Med Trop. 1998; 31(1):73-88.

9. Silva RA, Wanderley DMV, Domingos MF, Yasumaro S, Scandar SAS, Pauliquévis-Junior C, et al. Doença de Chagas: notificação de triatomíneos no Estado de São Paulo na década de 1990. Rev Soc Bras Med Trop. 2006;39(5): 488-94.

10. Wanderley DMV, Silva RA, Carvalho ME, Barbosa GL. Doença de Chagas: a vigilância entomológica no Estado de São Paulo. Bol Epidemiol Paulista (BEPA). 2007;4(38):10-4.

11. Brasil, Ministério da Saúde, Secretaria de Vigilância em Saúde. Consenso brasileiro em doença de Chagas. Rev Soc Bras Med Trop. 2005;38(Supl 3):7-29.

12. Gontijo ED, Dias JCP. Mortalidade por doença de Chagas em Minas Gerais: análise preliminar do período de 1978 a 1989. Rev Soc Bras Med Trop. 1994;27(Supl 2):119-20.

13. Santo AH. Tendência da mortalidade relacionada à varicela no Estado de São Paulo, Brasil, 1985 a 2004: estudo usando causas múltiplas de morte. Rev Panam Salud Publica. 2007;22(2):132-40.

14. Brasil. Lei 6 015, de 31 de dezembro de 1973. Diário Oficial da União 1973; 31 dez. Disponível em http:/ / www.planalto.gov.br/ CCIVIL/LEIS/L6015.htm. Acessado em 7 de dezembro de 2007.

15. Conselho Federal de Medicina. Resolução CFM n ${ }^{\circ}$ 1779/2005. Diário Oficial da União
2005; 05 dez: Seção I, p. 121. Disponível em http:/ / www.portalmedico.org.br/resolu coes/cfm/2005/1779_2005.htm. Acessado em 7 de agosto de 2007.

16. Brasil, Ministério da Saúde, Conselho Federal de Medicina, Centro Brasileiro de Classificação de Doenças. A declaração de óbito: documento necessário e importante. $2^{\mathrm{a}}$ ed. Brasília: Ministério da Saúde; 2007.

17. Brasil, Ministério da Saúde, Fundação Nacional de Saúde. Manual de Procedimentos do Sistema de Informações sobre Mortalidade. Brasília: Fundação Nacional de Saúde; 2001. Disponível em: http://bvsms.saude. gov.br/bvs/publicacoes/sis_mortalidade. pdf. Acessado em 24 de janeiro de 2008

18. Organização Mundial da Saúde. Classificação estatística internacional de doenças e problemas relacionados à saúde: $10^{\mathrm{a}}$ revisão. Vol. 2. Manual de instrução. São Paulo: Centro Colaborador da OMS para a Classificação de Doenças em Português/Edusp; 1993.

19. Santo AH. Causas múltiplas de morte: formas de apresentação e métodos de análise [tese]. São Paulo: Universidade de São Paulo; 1988.

20. Israel RA, Rosenberg HM, Curtin LR. Analytical potential for multiple cause-of-death data. Am J Epidemiol. 1986;124(2):161-79.

21. Santo AH. Potencial epidemiológico da utilização das causas múltiplas de morte por meio de suas menções nas declarações de óbito, Brasil, 2003. Rev Panam Salud Publica. 2007;22(3):178-86.

22. Santo AH, Pinheiro CE. Uso do microcomputador na seleção da causa básica de morte. Bol Ofic Sanit Panamer. 1995;119(4):319-27.

23. Santo AH, Pinheiro CE, Rodrigues EM. Comparative evaluation of underlying causes of death processed by the Automated Classification of Medical Entities and the Underlying Cause of Death Selection Systems. Rev Saude Publica. 1998;32(1):1-6

24. Santo AH. Avaliação da qualidade da codificação das causas de morte no Estado de São Paulo, Brasil. Inf Epidemiol SUS. 2000;9(3): 189-98.

25. Santo AH. Avaliação da codificação e do processamento das causas de morte pelo Sistema ACME no Estado de São Paulo, 1992 [tese de livre-docência]. São Paulo: Universidade de São Paulo; 1994

26. Pinheiro CE, Santo AH. Processamento de causas de morte em lote pelo sistema de seleção de causa básica. Rev Saude Publica. 1998;32(1):72-3.

27. Johansson LA, Westerling R. Comparing hospital discharge records with death certificates: can the differences be explained? J Epidemiol Community Health. 2002;56(4):301-8.

28. National Center for Health Statistics. Instruction Manual 2c: ICD-9 ACME decision tables for classifying the underlying causes of death, 1992. Hyattsville: National Center for Health Statistics; 1992.

29. National Center for Health Statistics. Instruction Manual 2c: ICD-10 ACME decision tables for classifying the underlying causes of death, 2008. Hyattsville: National Center for Health Statistics; 2008.

30. Centers for Disease Control and Prevention, National Center for Health Statistics. Compressed Mortality File 1999-2004. CDC WONDER On-line Database, compiled from Compressed Mortality File 1999-2004 Series 20 No. 2J, 2007. Disponível em http:// wonder.cdc.gov/cmf-icd10.html. Acessado em 11 de janeiro de 2008.

31. Organização Mundial da Saúde. Manual da classificação estatística internacional de doenças, lesões e causas de óbito: $9^{\mathrm{a}}$ revisão. Vol. 1. São Paulo: Centro da OMS para a Classificação de Doenças em Português/MS/ USP/OPAS; 1985

32. Organização Mundial da Saúde. Classificação estatística internacional de doenças e problemas relacionados à saúde. $10^{\mathrm{a}}$ revisão. Vol. 1 . São Paulo: Centro Colaborador da OMS para a Classificação de Doenças em Português/ Edusp; 1993.

33. Santo AH. Equivalência entre revisões da Classificação Internacional de Doenças: causas de morte. Rev Saude Publica. 2000;34(1): 21-8.

34. World Health Organization. International Classification of Diseases Translator: ninth and tenth revisions. Genebra: WHO; 1997.

35. Redelings MD, Wise M, Sorvillo F. Using multiple cause-of-death data to investigate associations and causality between conditions listed on the death certificate. Am J Epidemiol. 2007;166(1):104-8.

36. Santo AH, Pinheiro CE. Tabulador de causas múltiplas de morte. Rev Brasil Epidemiol. 1999;2(1/2):90-7.

37. Campbel H. Mortality analysis in time. Cohort analysis. Em: Manual of Mortality Analysis. Genebra: WHO; 1977. P. 109-14.

38. Litvoc J, Wanderley DMV, Camargo LMA. Mortalidade por doença de Chagas no Estado de São Paulo (Brasil): subsídios para o planejamento da assistência ao chagásico. Rev Saude Publica. 1992;26(2):59-65.

39. Drumond JAG, Marcopito LF. Migração interna e distribuição da mortalidade por doença de Chagas, Brasil, 1981/1998. Cad Saude Publica. 2006;22(10):2131-40.

40. Pereira MG. Características da mortalidade urbana por doença de Chagas, Distrito Federal, Brasil. Bol Oficina Sanit Panam. 1984;96 (3):213-21.

41. Rassi A Jr., Rassi A, Rassi SG. Predictors of mortality in chronic Chagas disease: a systematic review of observational studies. Circulation. 2007;115(9):1101-8.

42. Dias JCP. Chagas disease control and the natural history of human Chagas disease: a possible interaction? Mem Inst Oswaldo Cruz. 2000;95(Supl II):14-20.

43. Lima-Costa MF, Barreto SM, Guerra HL. Chagas' disease among older adults: branches or mainstream of the present burden of Trypanosoma cruzi infection? Int J Epidemiol. 2002;31(3):688-9. 
44. Lima-Costa MF, Peixoto SV, Giatti L. Tendências da mortalidade entre idosos brasileiros (1980-2000). Epidemiol Serv Saude. 2004; 13(4):217-28.

45. Prata A. Clinical and epidemiological aspects of Chagas disease. Lancet Infect Dis. 2001; 1(2):92-100.

46. Wanderley DMV, Litvoc J. Doença de Chagas como causa básica na região sudeste do Brasil: presença de causas contributárias. Rev Saude Publica 1994;28(1):69-75.

47. Carod-Artal FJ. Stroke: a neglected complication of American trypanosomiasis (Chagas' disease). Trans R Soc Trop Med Hyg. 2007; 101(11):1075-80.

48. Carod-Artal FJ, Ribeiro Lda S, Vargas AP. Awareness of stroke risk in chagasic stroke patients. J Neurol Sci. 2007;263(1-2):35-9.
49. Guariento ME, Orosz JEB, Gontijo JAR. Interação clínica entre moléstia de Chagas e hipertensão arterial primária em um serviço de referência ambulatorial. Arq Bras Cardiol. 1998;70(6):431-4.

50. Gurgel CB, Almeida EA. Frequency of hypertension in patients with chronic Chagas disease and its consequences on the heart: a clinical and pathological study. Arq Bras Cardiol. 2007;89(3):174-82.

51. França E, de Abreu DX, Rao C, Lopez AD. Evaluation of cause-of-death statistics for Brazil, 2002-2004. Int J Epidemiol. 2008;37(4): 891-901.

52. Brasil, Ministério da Saúde. Rede Interagencial de Informações para a Saúde. Indicadores e Dados Básicos - Brasil - 2006. Indicadores de Cobertura. Razão entre óbitos informados e estimados. Disponível em http://tabnet datasus.gov.br/cgi/idb2007/f11a.htm. Acessado em 31 de outubro de 2008.

53. Santo AH. Causas mal definidas de morte e óbitos sem assistência. Rev Assoc Med Bras. 2008;54(1):23-8.

Manuscrito recebido em 18 de novembro de 2008. Aceito em versão revisada em 19 de fevereiro de 2009.

ABSTRACT Objectives. To study mortality trends related to Chagas disease taking into account all mentions of this cause listed on any line or part of the death certificate.

\section{Chagas disease-related mortality trends, state of São Paulo, Brazil, 1985 to 2006: a study using multiple causes of death}

Methods. Mortality data for 1985-2006 were obtained from the multiple cause-ofdeath database maintained by the São Paulo State Data Analysis System (SEADE). Chagas disease was classified as the underlying cause-of-death or as an associated cause-of-death (non-underlying). The total number of times Chagas disease was mentioned on the death certificates was also considered.

Results. During this 22-year period, there were 40002 deaths related to Chagas disease: 34917 (87.29\%) classified as the underlying cause-of-death and $5085(12.71 \%)$ as an associated cause-of-death. The results show a $56.07 \%$ decline in the death rate due to Chagas disease as the underlying cause and a stabilized rate as associated cause. The number of deaths was $44.5 \%$ higher among men. The fact that $83.5 \%$ of the deaths occurred after 45 years of age reflects a cohort effect. The main causes associated with Chagas disease as the underlying cause-of-death were direct complications due to cardiac involvement, such as conduction disorders, arrhythmias and heart failure. Ischemic heart disease, cerebrovascular disorders and neoplasms were the main underlying causes when Chagas was an associated cause-of-death.

Conclusions. For the total mentions to Chagas disease, a $51.34 \%$ decline in the death rate was observed, whereas the decline in the number of deaths was only $5.91 \%$, being lower among women and showing a shift of deaths to older age brackets. Using the multiple cause-of-death method contributed to the understanding of the natural history of Chagas disease.

Key words Chagas disease; multiple causes of death; mortality; automatic data processing; Brazil. 